\title{
Isoflurane Impairs Low-Frequency Feedback but Leaves High-Frequency Feedforward Connectivity Intact in the Fly Brain
}

\author{
(1)Dror Cohen, ${ }^{1,3}$ Bruno van Swinderen, ${ }^{2}$ and ${ }^{-}$Naotsugu Tsuchiya ${ }^{1,3}$
}

\section{DOI:http://dx.doi.org/10.1523/ENEURO.0329-17.2018}

\begin{abstract}
${ }^{1}$ School of Psychological Sciences, Monash University, Melbourne 3800, Australia, ${ }^{2}$ Queensland Brain Institute, The University of Queensland, Brisbane 4072, Australia, and ${ }^{3}$ Monash Institute of Cognitive and Clinical Neuroscience, Monash University, Melbourne 3800, Australia
\end{abstract}

\begin{abstract}
Hierarchically organized brains communicate through feedforward (FF) and feedback (FB) pathways. In mammals, FF and FB are mediated by higher and lower frequencies during wakefulness. FB is preferentially impaired by general anesthetics in multiple mammalian species. This suggests FB serves critical functions in waking brains. The brain of Drosophila melanogaster (fruit fly) is also hierarchically organized, but the presence of FB in these brains is not established. Here, we studied FB in the fly brain, by simultaneously recording local field potentials (LFPs) from low-order peripheral structures and higher-order central structures. We analyzed the data using Granger causality (GC), the first application of this analysis technique to recordings from the insect brain. Our analysis revealed that low frequencies $(0.1-5 \mathrm{~Hz})$ mediated FB from the center to the periphery, while higher frequencies $(10-45 \mathrm{~Hz})$ mediated FF in the opposite direction. Further, isoflurane anesthesia preferentially reduced FB. Our results imply that the spectral characteristics of FF and FB may be a signature of hierarchically organized brains that is conserved from insects to mammals. We speculate that general anesthetics may induce unresponsiveness across species by targeting the mechanisms that support FB.
\end{abstract}

Key words: directed connectivity; Drosophila; feedback; general anesthesia; Granger causality; local field potentials

\section{Significance Statement}

In mammalian brains, neurons communicate through fast (high-frequency) feedforward (FF) and slow (low-frequency) feedback (FB) pathways. Various theories of consciousness propose that FB is crucial for the maintenance of consciousness. Here we used Granger causality (GC) analysis and multi-electrode recordings to investigate FB in the fly brain. Surprisingly, we found that the temporal characteristics of FF and FB are conserved in flies. Further, we found that isoflurane anesthesia preferentially reduced FB, consistent with results from mammalian studies. Our results imply that the mediation of FF and FB by higher and lower frequencies is a signature of hierarchically organized brains and that FB is a conserved, system-level target for general anesthetics.

\section{Introduction}

Many complex networks including brains are hierarchically organized. In hierarchical systems information trav- els both from the bottom to the top of the hierarchy, known as feedforward (FF), and from the top to the bottom of the hierarchy, known as feedback (FB). It is only 
recently that the dynamic characteristics of neural FB and FF have been reported. For example, studies that investigated directed connectivity using Granger causality (GC) across the awake monkey visual hierarchy have shown that the alpha/beta and gamma bands preferentially mediate FB and FF, respectively (Bastos et al., 2014; van Kerkoerle et al., 2014). This pattern of low-frequency FB/ high-frequency FF has also been reported across the visual (Michalareas et al., 2016) and auditory system (Fontolan et al., 2014) in awake humans. These studies imply that FB is mediated by lower frequencies than those that mediate FF.

The difference in frequency bands that mediate FB and FF is consistent with the functional explanation of these under the hierarchical predictive coding framework (Hohwy, 2013). According to predictive coding, compared to FF, FB necessarily operates at slower timescales because its main function is to provide prediction of incoming signals through the incorporation of memory and expectation. On the other hand, FF has to react to quickly changing sensory inputs, necessarily operating at faster timescales than FB. Note that these hypotheses may be true for all hierarchically organized biological brains, from insects to mammals, to the extent that these functional requirements are shared across species.

Anatomically, fly brains are hierarchically organized from peripheral sensory layers to central nuclei, which are responsible for more abstract computation (Paulk et al., 2013a; Shih et al., 2015; Haberkern and Jayaraman, 2016). Like mammals, wakeful insect brains also face functional challenges in adjusting fast sensory inputs based on prior experience on a slower time scale (Card and Dickinson, 2008; Combes, 2015; Kim et al., 2015; Mischiati et al., 2015). In addition, recent work suggests that the topological organization of the mammalian brain, which shares a number of similarities with the topological organization of the fly brain (Shih et al., 2015), influences the direction of information flow (Moon et al., 2015; Mejias et al., 2016; Moon et al., 2017). This evidence suggests that similar dynamic characteristics of FF and FB processing will be present in the insect brain.

A separate line of inquiry suggests that FB in particular is crucial for waking brain functions. A number of studies have demonstrated that general anesthetics preferentially

N.T. was funded by the Australian Research Council Future Fellowship FT120100619 and the Discovery Project DP130100194. B.v.S. was funded by National Health and Medical Research Council Project APP1103923. The project was made possible through the support of a grant (TWCF0199) from Templeton World Charity Foundation, Inc. This work was also supported by computational resources provided by the Australian Government through MASSIVE under the National Computational Merit Allocation Scheme.

Acknowledgments: We thank Jakob Hohwy and Yuri Saalmann for helpful comments on this manuscript.

Correspondence should be addressed to either of the following: Naotsugu Tsuchiya, 770 Blackburn Road, Clayton 3168, Victoria, Australia, E-mail: naotsugu.tsuchiya@monash.edu; or Dror Cohen, 770 Blackburn Road, Clayton 3168, Victoria, Australia, E-mail: dror.cohen@monash.edu.

DOI:http://dx.doi.org/10.1523/ENEURO.0329-17.2018

Copyright (C) 2018 Cohen et al.

This is an open-access article distributed under the terms of the Creative Commons Attribution 4.0 International license, which permits unrestricted use, distribution and reproduction in any medium provided that the original work is properly attributed. reduce FB from frontal to posterior areas as measured using human scalp level electroencephalography (EEG; Lee et al., 2009; Ku et al., 2011; Boly et al., 2012; Jordan et al., 2013; Lee et al., 2013; Ranft et al., 2016). Although a subset of studies show differing results (Barrett et al., 2012; Nicolaou et al., 2012; Maksimow et al., 2014), the disrupted frontal-parietal connectivity has also been demonstrated using functional magnetic resonance imaging (fMRI; Hudetz and Mashour, 2016). In addition, general anesthetic suppression of FB has also been reported in rodents (Imas et al., 2006; Pal et al., 2016), ferrets (Wollstadt et al., 2017), and monkeys (Lamme et al., 1998; Papadopoulou et al., 2015). Note, however, that Wollstadt et al. (2017) show that FB (prefrontal cortex to V1) is reduced, but that this reduction may be a result of reduced "source information" within the prefrontal cortex. Also, Lamme et al. (1998) have shown that general anesthesia reduces contextual processing, which has been taken as an indirect support for the impairment of FB influences to V1.

Flies are also rendered unresponsive by general anesthetics and in similar concentrations to humans (van Swinderen and Kottler, 2014; Zalucki and van Swinderen, 2016). Indeed, the cellular and molecular machinery that makes up the nervous system is largely conserved across species (Littleton and Ganetzky, 2000), such that the cellular and molecular targets of general anesthetics are also likely to be conserved (Sonner, 2008; van Swinderen and Kottler, 2014; Zalucki and van Swinderen, 2016). Whether the conserved cellular and molecular targets of general anesthetics also translate to conserved system-level general anesthetic effects, such as reduced FB, is currently unknown.

Here, we investigated the dynamic characteristics of FB and FF in the small brains of fruit flies, Drosophila melanogaster. We analyzed directional connectivity in LFPs recorded from low-order peripheral structures and higherorder central structures. This showed that low frequencies (0.1-5 Hz) mediated FB from the center to the periphery, while higher frequencies $(10-45 \mathrm{~Hz})$ mediated FF in the opposite direction. Further, the general anesthetic isoflurane reduced FB while leaving FF intact. Our results imply that the preferential mediation of FB and FF by low and high frequencies, respectively, may be a signature of hierarchically organized brains and that general anesthetics induce unresponsiveness by targeting FB mechanisms.

\section{Materials and Methods}

\section{Overview}

We analyzed local field potentials (LFPs) data previously recorded from the brains of awake and anesthetized flies (Cohen et al., 2016). Whereas Cohen et al. (2016) focused on visually evoked responses, here we focus on the analysis of power, coherence, and GC of spontaneous LFPs, and how these are affected by isoflurane. In this section, we briefly recap the experimental setup. For the full experimental details, see Cohen et al. (2016).

Thirteen female laboratory-reared $D$. melanogaster (Canton $S$ wild type) flies (3-7 d after eclosion) were collected 


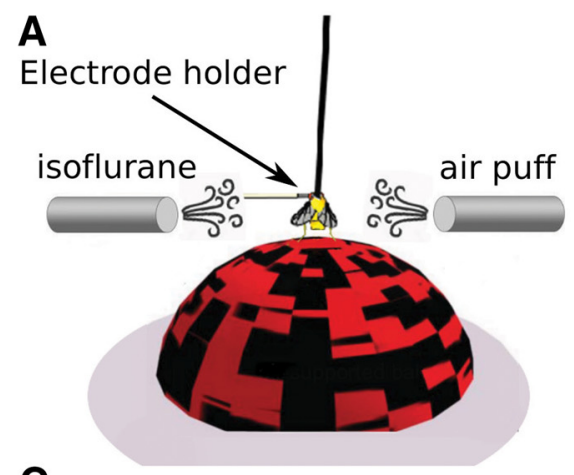

C
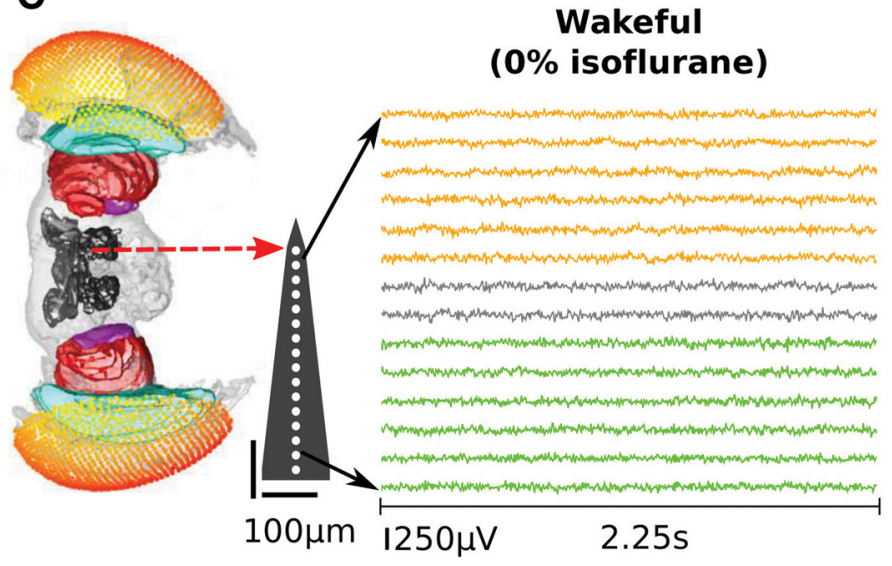

D

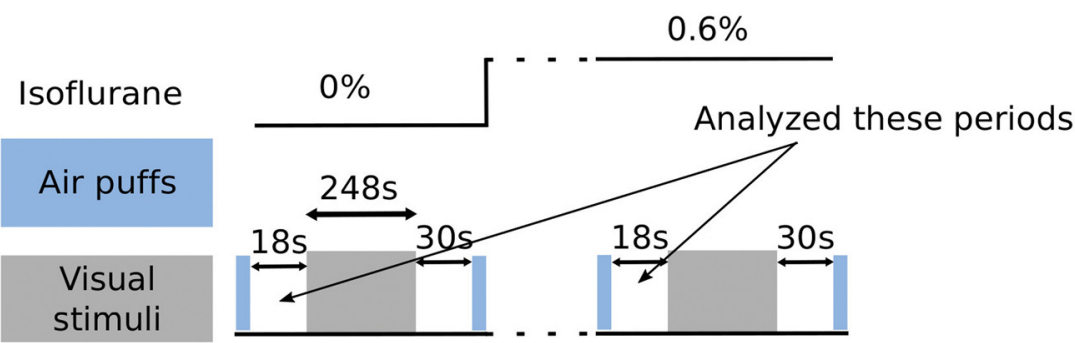

B

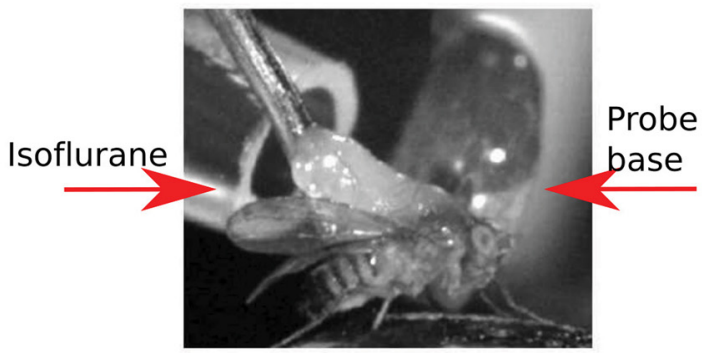

\section{Anesthetized (0.6\% isoflurane)}

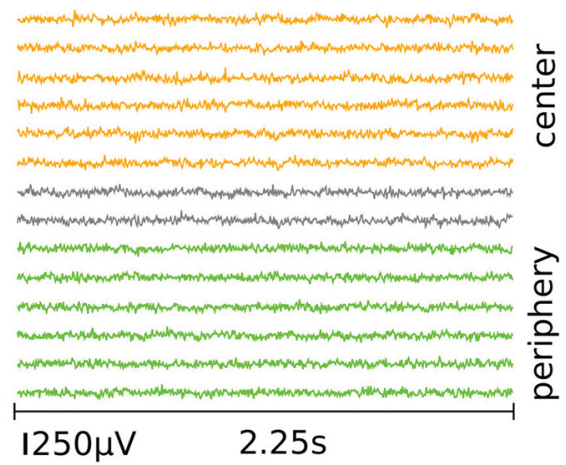

Figure 1. Experimental setup. A, Flies were dorsally fixed to a tungsten rod and placed on an air-supported ball where they could freely walk. Isoflurane in different volumetric concentrations was delivered through a rubber hose. Air puffs were used to gauge flies' responsiveness. A 16-contact electrode probe mounted on an electrode holder was inserted laterally from the left into the fly's eye. Only the electrode holder is visible at the depicted scale. B, A close-up view contralateral to the electrode insertion site showing the tethered fly, isoflurane delivery hose, and probe base. $\boldsymbol{C}$, Example of pre-processed LFPs data before anesthesia (0\% isoflurane) from one fly. A standardized fly brain is shown for comparison (Paulk et al., 2013b, 2015). The electrode contacts are indicated by white dots (not to scale). For visualization the LFPs are shown subsampled to $250 \mathrm{~Hz}$ (analyzed at $1000 \mathrm{~Hz}$ ). Six channels are grouped as peripheral, corresponding to the optic lobe (green), and another six channels are grouped as central, corresponding to the central brain (orange). $\boldsymbol{D}$, An experiment consisted of multiple blocks at different concentration of isoflurane (top black line). Each block proceeded with (1) a series of air puffs (light blue rectangles) followed by $18 \mathrm{~s}$ of rest; (2) presentation of visual stimuli (248 s); (3) 30 $\mathrm{s}$ of rest followed by a series of air puffs; and (4) isoflurane concentration change followed by $180 \mathrm{~s}$ of rest.

under cold anesthesia, tethered and positioned on an air-supported Styrofoam ball. Linear silicon probes with 16 electrodes separated by $25 \mu \mathrm{m}$ (Neuronexus 3mm-25177) were inserted laterally to the eye of the fly until the most peripheral electrode site was just outside the eye. This probe covers approximately half of the fly brain and records neural activity from both peripheral and central brain structures (Fig. 1C). A fine tungsten wire was inserted in the thorax and used as a reference electrode.

Isoflurane was delivered through a rubber hose connected to an evaporator (Fig. $1 A, B$ ). The effects of isoflu- rane were tested at concentrations of $0 \%$ and $0.6 \%$ (for details, see Cohen et al., 2016). An olfactory stimulus controller was used to deliver six air puffs to gauge the behavioral responsiveness of the flies in each concentration of isoflurane. Fly movement activity in response to the air puff was recorded with a camera. We used the video data to confirm that $0.6 \%$ isoflurane abolished the behavioral responsiveness and that behavioral responsiveness returned after recovery (Cohen et al., 2016).

An experiment consisted of several blocks, each at a different concentration of isoflurane. Each block started 
with the delivery of a series of air puffs, followed by $\sim 18$ $s$ of rest, which is the critical period of data for this paper. Visual stimuli were presented, lasting $248 \mathrm{~s}$ in total, followed by an additional $30 \mathrm{~s}$ of rest. A series of air puffs were delivered again and then the isoflurane concentration was changed. After $180 \mathrm{~s}$ of adjustment to the new isoflurane concentration the experimental block was repeated (Fig. 1D).

In Cohen et al. (2016), we focused on the analysis of the visually-evoked data, which corresponds to the $248 \mathrm{~s}$ period of visual stimuli presentation. Here we focused on an 18-s period before the presentation of visual stimuli (Fig. 1D).

This no-visual stimulation period is better suited for coherence and GC analysis than the visual stimulation period because the stimulus-evoked responses can result in erroneous interpretation of coherence and GC (Truccolo et al., 2002; Wang et al., 2008). We focused on the period before rather than after visual stimuli presentation because we reasoned the latter period may also reflect residual visual activity.

The datasets analyzed in this study are available from the corresponding authors on request.

\section{LFP pre-processing}

LFPs were recorded at $25 \mathrm{kHz}$, downsampled to 1000 $\mathrm{Hz}$, and the most peripheral electrode site was removed from the analysis. We rereferenced the remaining 15 unipolar channels by subtracting adjacent channels (bipolar rereferencing) to obtain a set of 14 differential signals which we refer to as "channels" throughout this paper (Bastos et al., 2014; Trongnetrpunya et al., 2016; Cohen and Tsuchiya, 2017).

We divided the 18-s period into eight consecutive epochs of $2.25 \mathrm{~s}$. We removed line noise from each epoch and bipolar rereferenced channel using three tapers, a window of $0.75 \mathrm{~s}$ and a step size of $0.375 \mathrm{~s}$ using the rmlinesmovingwinc.m function from the Chronux toolbox (http://chronux.org/; Mitra and Bokil, 2007). We then linearly detrended and $z$-scored each channel and epoch by removing the mean and dividing by the standard deviation across temporal points within each epoch (Seth, 2010; Bastos et al., 2014). Figure $1 C$ shows example of the resulting LFPs from one fly before ( $0 \%$ isoflurane) and during anesthesia ( $0.6 \%$ isoflurane).

\section{Analyzing power}

For power analysis we linearly detrended the preprocessed LFPs without the $z$-scoring operation, because $z$-scoring obscures overall shifts in power when comparing pre- and postanesthesia. We calculated power for each pre-processed channel $i(\mathrm{i}=[1-14])$, epoch e (1-8), $k \%$ isoflurane concentration ( $\mathrm{k}=[0$ (air), 0.6]) and frequency $\omega, S_{e k}^{i}(\omega)$ over the 2.25-s epochs. Using three tapers, the half bandwidth was $0.89 \mathrm{~Hz}$ (Mitra and Pesaran, 1999). We further averaged power across eight epochs to obtain one estimate of power for each channel and isoflurane concentration $S_{k}^{i}(\omega)$.

We analyzed the average power across channels within the center $S_{k}^{C}(\omega)$ and periphery $S_{k}^{P}(\omega)$ in units of $10 \log 10 \mu \mathrm{V}^{2}$, following the division of channels into peripheral chan- nels $=[1-6]$ and central channels $=[9-14]$ as in Cohen et al . (2016)

$$
\begin{aligned}
& S_{k}^{P}(\omega)=\frac{1}{6} \sum_{i=1}^{6} S_{k}^{i}(\omega) \\
& S_{k}^{C}(\omega)=\frac{1}{6} \sum_{i=9}^{14} S_{k}^{i}(\omega)
\end{aligned}
$$

In the fly brain, the locations of the peripheral channels correspond to the primary visual systems while those of the central channels to higher-order structures (Paulk et al., 2013a; Shih et al., 2015; Haberkern and Jayaraman, 2016). In Cohen et al. (2016), we found that these two rough divisions explained different electrophysiological responses during the visual stimulation period.

We report the effect of isoflurane on power in the central brain as the difference in power between $0.6 \%$ and $0 \%$ in decibels $(\mathrm{dB})$

$$
\Delta S_{0.6}^{C}(\omega)=S_{0.6}^{C}(\omega)-S_{0}^{C}(\omega)
$$

Analogous derivations give the effect of $0.6 \%$ isoflurane on power in the periphery $\Delta S_{0.6}^{P}(\omega)$.

Previous work has shown that the fly heartbeat can manifest as low-frequency $(2-4 \mathrm{~Hz})$ oscillatory activity in central channels (Paulk et al., 2013b; Yap et al., 2017). Heartbeats are unlikely to affect the results presented here because we use bipolar rereferencing which eliminates common input to neighboring channels, as may be expected from a muscle artifact (Cohen and Tsuchiya, 2017). To further rule out heartbeat as a potential confound we also analyzed GC (see Non-parametric GC analysis) in our data after excluding three flies in which we detected potential heartbeat in central channels by visual inspection of the power spectra. The results of this analysis closely matched those obtained with all thirteen flies, indicating the any heartbeat contamination is unlikely to affect our results.

\section{Analyzing coherence}

Coherence measures the strength of linear dependency between two signals in the frequency domain (Bendat and Piersol, 2000). It is defined as

$$
C^{i j}(\omega)=\frac{a b s\left(C S^{i j}(\omega)\right)^{2}}{S^{i}(\omega) S^{j}(\omega)}
$$

$\operatorname{CS}^{i j}(\omega)$ is the cross-spectrum between channels $\mathrm{i}$ and $\mathrm{j}$, and $S^{i}(\omega)$ and $S^{j}(\omega)$ are the power spectra of channels $i$ and j, respectively. To estimate coherence for each bipolar rereferenced channel pair $i$ and $j(i, j=[1-14])$, epoch $e$ $(1-8)$ and $k \%$ isoflurane concentration ( $\mathrm{k}=[0$ (air), 0.6]) we used the multitaper method with nine tapers for each 2.25-s epoch, giving a half bandwidth of $2.22 \mathrm{~Hz}$ (Mitra and Pesaran, 1999). The auto and cross spectra for channels $i$ and $j$ at isoflurane concentration $k \%$ were averaged across the eight epochs to give one estimate of coherence at each isoflurane concentration.

We report center $\left(C^{C}\right)$ and periphery $\left(C^{P}\right)$ coherence as averaged across all non-neighboring center and periphery channel pairs 


$$
\begin{aligned}
& C_{k}^{C}(\omega)=\frac{1}{10} \sum_{i=9}^{12} \sum_{j=i+2}^{14} C_{k}^{i j}(\omega) \\
& C_{k}^{P}(\omega)=\frac{1}{10} \sum_{i=1}^{4} \sum_{j=i+2}^{6} C_{k}^{i j}(\omega)
\end{aligned}
$$

We exclude neighboring channels pairs because these show artefactual high coherence due to the bipolar rereferencing (for details, see Shirhatti et al., 2016; Cohen and Tsuchiya, 2017).

We report center-periphery coherence $\left(C^{C P}\right)$ as averaged across all center-periphery pairs

$$
C_{k}^{C P}(\omega)=\frac{1}{36} \sum_{i=1}^{6} \sum_{j=9}^{14} C_{k}^{i j}(\omega)
$$

\section{Estimating the coherence bias}

Because coherence is limited to the $0-1$ range it has an inherent positive bias (Jarvis and Mitra, 2001). We used surrogate data statistics to estimate the empirical coherence bias in our data. The advantages of this method are (1) that it provides an empirical lower bound above which coherence may be considered significant and (2) that it accounts for potentially frequency-specific coherence biases sometimes observed for two time-series with similar power spectra (Faes et al., 2004).

For each of 13 flies we created surrogate data in which we randomized the phase of the signal while keeping the amplitude the same (Faes et al., 2004; Fasoula et al., 2013). We performed this 200 times for each channel (1-14), epoch (1-8) and isoflurane concentration ([0\%, $0.6 \%]$ ) resulting in surrogate data with the same power spectra as the original data but with near-zero cross spectra between channels. The surrogate data describes the null hypothesis that there is no coherence in the data.

To estimate the center, periphery and center-periphery coherence bias at $k \%$ isoflurane we calculated coherence as described above for each of the 200 surrogate data sets, and averaged the estimates across all 200 surrogates. We obtained the $95 \%$ confidence intervals by determining the 2.5 and 97.5 percentiles across the 200 surrogates for each fly.

When we compared the coherence bias in $0.6 \%$ and $0 \%$ isoflurane, we observed differences in some frequencies $(0.1-5 \mathrm{~Hz})$, but the magnitudes of the differences were very small (largest difference was 0.0038 at around $11 \mathrm{~Hz}$ for center coherence). Accordingly, our coherence results in $0.6 \%$ and $0 \%$ isoflurane with or without bias correction were nearly identical.

\section{Non-parametric GC analysis}

In simple terms, a signal $X$ is said to Granger-cause a signal $Y$ if past values of $X$ improve predictions of future values of $Y$. This notion of causality is based on modeling $X$ and $Y$ as autoregressive processes. GC can be estimated in either the time or frequency domain. The latter provides spectral decomposition of GC influences. We hypothesized that examining GC influences at different frequencies is critical because recent work that studied mammalian brains showed that different directional interactions were mediated by different frequencies (Bastos et al., 2014; van Kerkoerle et al., 2014; Michalareas et al., 2016).

GC can be estimated parametrically by fitting an autoregressive model to the data (Geweke, 1982, 1984; Chen et al., 2006). The drawback of this approach is that it requires selection of the model order. Alternatively, GC can be non-parametrically estimated directly from the spectral density matrix (Dhamala et al., 2008; Wen et al., 2013). The spectral density matrix for channels $i$ and $j$ at isoflurane concentration $k \% \mathbf{Q}_{k}^{j i}(\omega)$ is obtained by setting the diagonal elements to the auto-spectra and the crossdiagonal elements to the cross-spectra (Wen et al., 2013)

$$
\mathbf{Q}_{k}^{i j}(\omega)=\left(\begin{array}{cc}
S_{k}^{i}(\omega) & C S_{k}^{i j}(\omega) \\
C S_{k}^{i j}(\omega) & S_{k}^{i}(\omega)
\end{array}\right)
$$

We estimated the spectral density matrix using the same estimates of auto- and cross-spectra as those used for coherence analysis. To obtain Granger-causal influences from channel $i$ to channel $j$ at isoflurane concentration $k \% f_{k}^{i \rightarrow j}(\omega)$ we first factorized the spectral density matrix using the sfactorization_wilson.m function and then estimated the Granger-causal influences using the ft_connectivity_Granger.m function. These functions are available from the FieldTrip toolbox (Oostenveld et al., 2011).

\section{Grouping FF and FB influences}

In the fly brain, visual information enters through the retina and is sequentially processed by the lamina, medulla and lobula, collectively referred to as the optic lobe (Sanes and Zipursky, 2010; Paulk et al., 2013a). Visual projection neurons (VPNs) convey information from lower to higher-order visual processing centers, called optic glomeruli, which are located more centrally (Otsuna and Ito, 2006). Relatively little is known about computations in the optic glomeruli, but recent studies suggest a role in second-order motion processing (Zhang et al., 2013) and processing that mediates natural avoidance behaviors (Wu et al., 2016). Specific optic glomeruli, such as the ventrolateral protocereberum (Ito et al., 2014), send projections back to the lobula and medulla, as well as reciprocated projections to other central structures (Otsuna and Ito, 2006; Shih et al., 2015). Our simple summary of this connectome is that visual information enters in the periphery and sequentially conveyed through FF connections toward more central optic glomeruli, which convey FB back to the optic lobe.

We emphasize that our definition of FF and FB is based only on this anatomic organization. Because we did not manipulate bottom-up or top-down processing through stimulus characteristics or task demands, we do not claim that our FF and FB estimates directly reflect bottom-up sensory processing or top-down cognitive functions.

To estimate overall FF GC influences at $\mathrm{k} \%$ isoflurane $f_{k}^{F F}(\omega)$ we averaged across all GC influences $f_{k}^{i \rightarrow j}(\omega)$ from the periphery to the center 


$$
f_{k}^{F F}(\omega)=\frac{1}{36} \sum_{i=1}^{6} \sum_{j=9}^{14} f_{k}^{j \rightarrow i}(\omega)
$$

where channel 1 is the most peripheral and channel 14 is the most central. Analogous derivations give FB GC influences

$$
f_{k}^{F B}(\omega)=\frac{1}{36} \sum_{i=1}^{6} \sum_{j=9}^{14} f_{k}^{j \rightarrow i}(\omega)
$$

\section{Estimating the Granger causal influences bias}

GC influences are always positive and therefore have a positive bias. We checked whether the GC bias differed between FF and FB or across isoflurane concentrations. To estimate the GC bias, we used the same procedure for the estimation of the coherence bias through phase randomization. This procedure results in 200 estimates of FF and FB GC influences for each fly, at each isoflurane concentration $k \%$. We obtained the final estimate of the FF and FB GC bias by averaging across all 200 surrogates.

The differences between FF and FB GC biases at each isoflurane concentration, as well as the differences in FF and $\mathrm{FB}$ GC biases between isoflurane concentrations were negligible. The maximum difference in group average bias was 0.0008 (difference between FF and FB at $0.6 \%$ isoflurane at around $5 \mathrm{~Hz}$ ). The difference in $\mathrm{GC}$ bias averaged across $0.1-45 \mathrm{~Hz}$ were all negligible: 0.00006 (between FF and FB in 0\% isoflurane), 0.0002 (between FF and FB in $0.6 \%$ isoflurane), 0.0002 (between $0 \%$ and $0.6 \%$ isoflurane for $\mathrm{FF}$ ), and 0.0001 (between $0 \%$ and $0.6 \%$ isoflurane for $\mathrm{FB}$ ).

\section{Directed asymmetry index (DAl) analysis}

Because the magnitude and variance of GC influences are dependent on the frequencies, some form of normalization is necessary for statistically comparing them. For this purpose, we adopt the DAl (Lee et al., 2013; Bastos et al., 2015; Michalareas et al., 2016), which is defined as

$$
D A I=\frac{F F-F B}{F F+F B}
$$

The numerator of the DAl captures the predominant direction of the GC influence. The denominator provides normalization for the total GC influences at a given frequency. DAl allows comparisons across conditions and frequencies where there may be an overall net increase or decrease in GC influences.

Given our enumeration of channels (i.e., $i->j$ indicates FF influences if $i<j$ ), the DAl between channel $i$ and $j$ at isoflurane concentration $k \%$ is given by

$$
D A l_{k}^{i}(\omega)=\frac{f_{k}^{i \rightarrow j}(\omega)-f_{k}^{j \rightarrow i}(\omega)}{f_{k}^{i \rightarrow j}(\omega)+f_{k}^{j \rightarrow i}(\omega)}, i \in[1-6], j \in[9-14],
$$

We report the DAl averaged over all center-periphery channel pairs

$$
D A I_{k}(\omega)=\frac{1}{36} \sum_{i=1}^{6} \sum_{j=9}^{14} D A I_{k}^{i j}(\omega)
$$

\section{Statistical analysis}

To test for statistical differences between $0 \%$ and $0.6 \%$ isoflurane across frequencies (power, coherence, GC, and DAl spectrums) we used randomization-based, non-parametric statistical tests with cluster-based multiple comparison correction (Maris and Oostenveld, 2007). Each of the 13 flies provided one spectrum for each condition ( $0 \%$ and $0.6 \%$ isoflurane). We then performed paired t tests across 13 flies at each frequency to obtain $t$ scores. If we found a cluster of continuous frequencies at $p<0.05$ level, then we treated them as a first-level significant cluster. As a second-level statistic, we used the sum of the $t$ scores across frequencies within each cluster. We then preformed 8191 randomizations corresponding to all possible randomizations of thirteen flies across the two conditions $\left(2^{-13}-1=8191\right)$ and calculated the second-level statistic for each randomization. From each randomization, the largest second-level statistics was chosen and used for constructing a randomized distribution. We compared the observed second-level statistics against the randomized distribution to obtain $p$ values.

Figures 2-4 show the results from the first-level (uncorrected) $t$ tests as well as the second-level cluster-based statistics, which corrects for multiple comparisons across frequencies. When we compared DAl spectrums against zero (Fig. $4 B, F, J$ ), we randomized the $\mathrm{FF}$ and $\mathrm{FB}$ labels for each fly (Michalareas et al., 2016). For testing whether the DAls averaged across low and high frequencies were different from zero (Fig. 4C,D,G,H) we randomized the FF and $F B$ labels and used the group mean as the test metric. For testing the effect of isoflurane on the DAls averaged across low and high frequencies (Fig. $4 K, L$ ), we randomized the isoflurane concentrations labels and used the group mean difference between the conditions as the test metric.

\section{Results}

\section{Isoflurane reduces spontaneous power in the center and periphery}

We investigated the dynamic characteristics of FB and FF and how these are affected by anesthesia by analyzing LFPs recorded from the center and periphery of awake and anesthetized fly brains (Fig. 1). Before analyzing FB and FF we first assessed whether neural activity is distinguishable across the center and periphery. To do this we analyzed the power of central and peripheral channels separately (Fig. 2A). We found that power in both the center and periphery was highest at low frequencies and decreased as frequency increased. In low frequencies, power in the center was significantly greater than power in the periphery, demonstrating that neural activity was indeed distinguishable across these two anatomic areas (Fig. 2A). At a concentration of $0.6 \%$ isoflurane, the flies were rendered unresponsive, as probed by air puffs (Cohen et al., 2016). We found that during anesthesia, power in both the center and periphery was reduced $(0.6 \%$ 


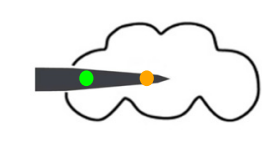

\section{Wakeful ( $0 \%$ isoflurane)}

A

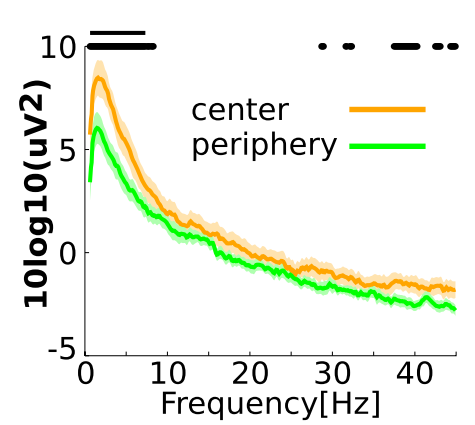

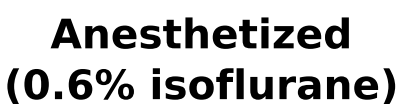

\section{Anesthetized-Wakeful (0.6\%-0\% isoflurane)}

B

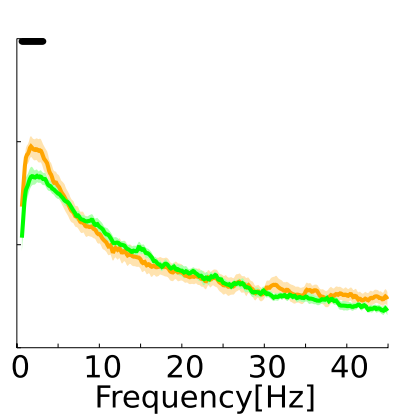

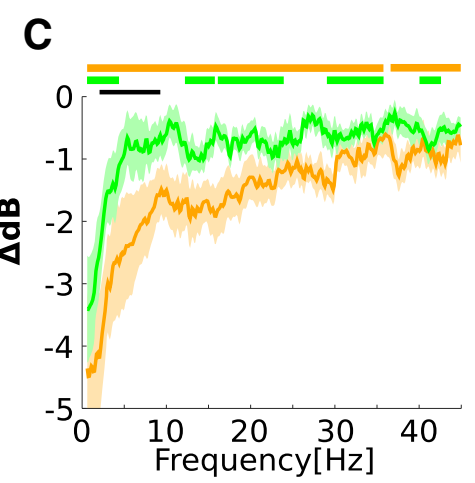

Figure 2. Isoflurane reduces power in the center and periphery. A, Group average power in the center (orange) and periphery (green) in $0 \%$ isoflurane (before any anesthesia). A schematic representation of the fly head and inserted electrode showing the estimated positions of one central (orange) and one peripheral (green) channel is depicted above. Black dots and solid line mark uncorrected and corrected significant differences between center and periphery power at the $p<0.05$ level, respectively (see Materials and Methods). $\boldsymbol{B}$, Same as $\boldsymbol{A}$ in $0.6 \%$ isoflurane. $\boldsymbol{C}$, The effect of isoflurane on power in the center and periphery, obtained by subtracting values in $0 \%$ isoflurane from values in $0.6 \%$ isoflurane. Negative values indicate that isoflurane reduced power for both the center and periphery. For central channels, orange thick lines above indicate significant reductions due to isoflurane (corrected, $p<0.05$ ). For peripheral channels, green thick lines above indicate corresponding significant reductions (corrected, $p<0.05$ ). Greater reductions in power in the center compared to the periphery are indicated by the black line (corrected, $p<0.05)$. Shaded area represents SEM across flies $(N=13)$ for all panels.

isoflurane; Fig. 2B). For the center, power was significantly reduced across almost all frequencies tested. For the periphery the reduction was smaller and only significant in a subset of the frequencies tested (Fig. 2C). The reduction in power in the center was greater than the periphery for low frequencies. These observations are consistent with an overall suppression of neural activity but also suggest that this suppression is more consistent in the higher-order central structures of the fly brain.

\section{Isoflurane reduces center, periphery, and center- periphery coherence}

Next, we assessed the effects of isoflurane on local and global processing. To do this, we analyzed coherence, a spectral measure of linear dependency between signals (Bendat and Piersol, 2000). To assess the effects on local processing, we analyzed coherence within the center and periphery. We calculated center and periphery coherence using all non-neighboring center and periphery channel pairs (for details, see Materials and Methods).

Within-center coherence was highest for low frequencies and showed an additional peak around $10-20 \mathrm{~Hz}$ (Fig. 3A). Because coherence is confined to $0-1$, it is inherently biased. Using surrogate data methods (see Materials and Methods), we found that the coherence was above the bias level for $0.1-45 \mathrm{~Hz}$ (Fig. $3 A$ ). We repeated the coherence analysis during anesthesia and found that the coherence remained above the bias for all frequencies (Fig. 3B). Isoflurane significantly reduced within-center coherence around $15 \mathrm{~Hz}$ (Fig. 3C).
Similarly, within-periphery coherence was highest for low frequencies, decreased as frequency increased and remained above the bias for $0.1-45 \mathrm{~Hz}$ (Fig. 3D). Unlike withincenter coherence, however, within-periphery coherence had no prominent peak in the $10-20 \mathrm{~Hz}$ range (Fig. 3D). During anesthesia all frequencies remained above the bias (Fig. 3E). Isoflurane significantly reduced within-periphery coherence in the $15-25 \mathrm{~Hz}$ range (Fig. 3F).

Finally, we assessed the effects of anesthesia on more global processing by calculating center-periphery coherence, which we calculated using all center-periphery channel pairs (for details, see Materials and Methods). We found that similar to center and periphery coherence, center-periphery coherence was highest for low frequencies, decreased with increasing frequency and remained above the bias for $0.1-45 \mathrm{~Hz}$ (Fig. 3G). During anesthesia, low-frequency coherence remained above the bias, whereas high-frequency coherence was not distinguishable from the bias (Fig. $3 H$ ). Isoflurane significantly reduced center-periphery coherence around the $10-25 \mathrm{~Hz}$ range.

In sum, our coherence results indicate that isoflurane impaired localized processing within the center and periphery as well as global processing between the center and periphery.

Low- and high-frequencies preferentially mediate FB and $\mathrm{FF}$, respectively

Finally, we analyzed directional connectivity. To dissect the directionality we used GC analysis (Dhamala et al., 


\section{Wakeful \\ Anesthetized ( $0 \%$ isoflurane) \\ (0.6\% isoflurane)}

A

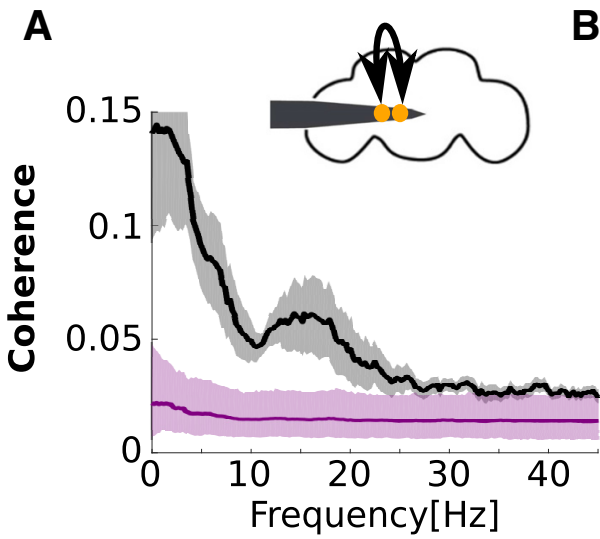

D

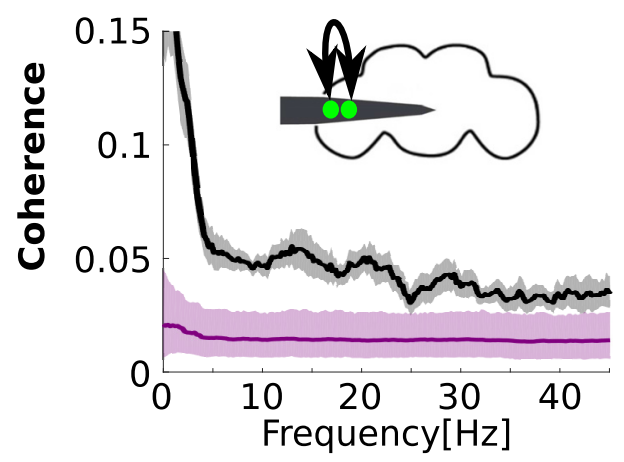

G

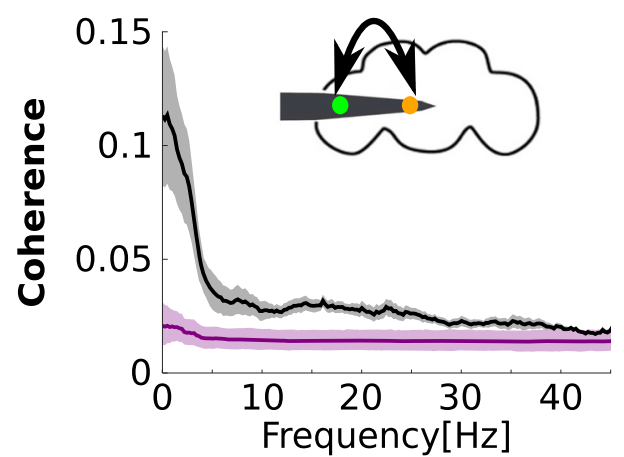

B

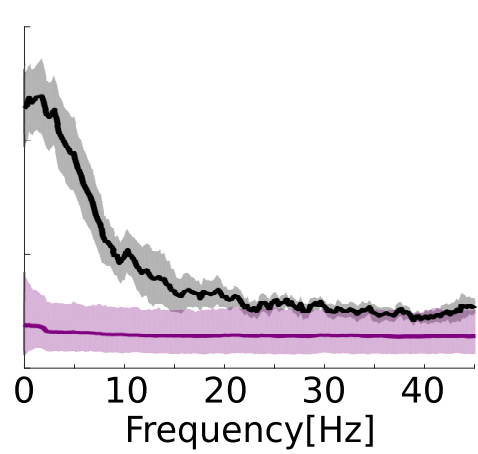

E

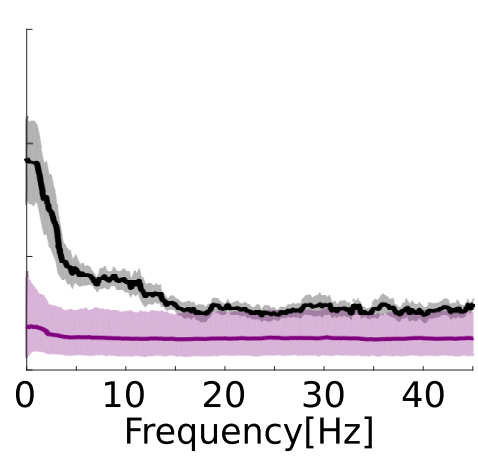

H

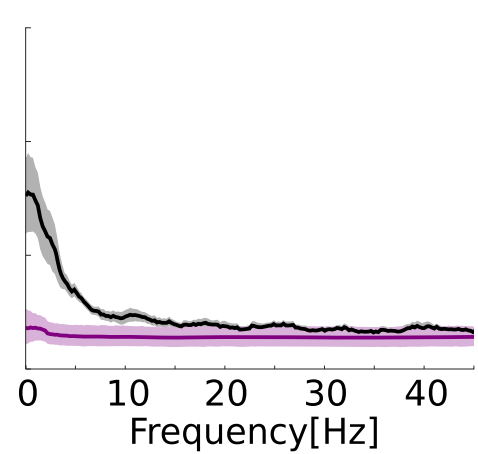

\section{Anesthetized-Wakeful (0.6\%-0\% isoflurane)}

C 0.04

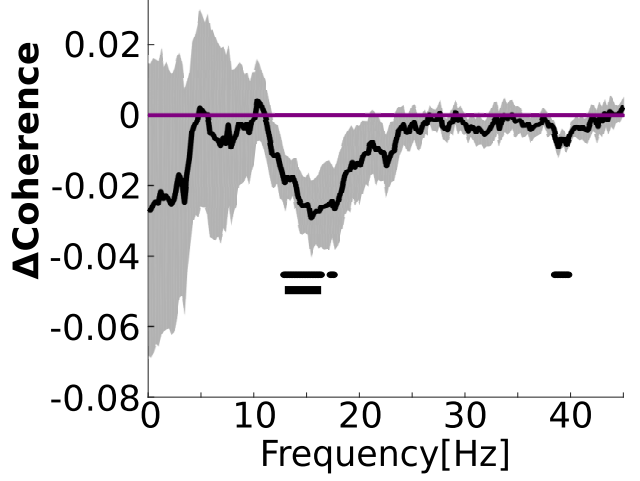

F 0.02

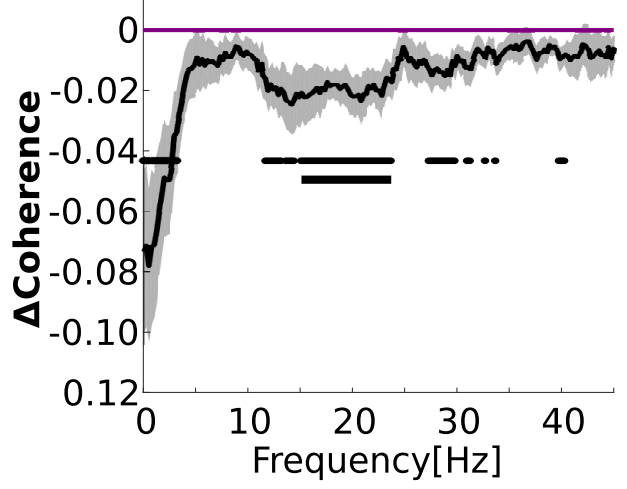

I

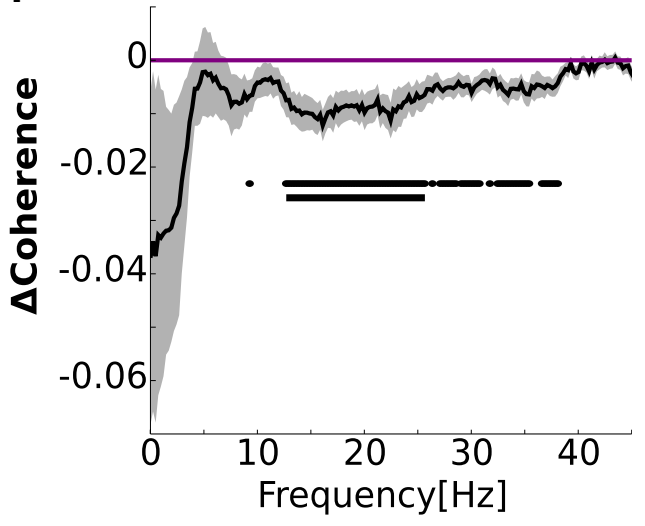

Figure 3. The effect of isoflurane on center, periphery, and center-periphery coherence. $\boldsymbol{A}$, Coherence between electrodes in the central brain in $0 \%$ isoflurane (black line). Gray shades represent SEM across flies $(N=13)$. Purple line and shades represents the bias level and its $95 \%$ confidence intervals. A schematic representation of the fly head and inserted electrode showing the estimated positions of two central channels is depicted above. $\boldsymbol{B}$, Same as a in $0.6 \%$ isoflurane. $\boldsymbol{C}$, The effect of isoflurane on center coherence, obtained by subtracting values in $0 \%$ isoflurane from values in $0.6 \%$ isoflurane. Dots and solid lines below the effect of isoflurane (C, $\boldsymbol{F}, \boldsymbol{I})$ indicate significant reductions $(p<0.05)$ uncorrected and corrected, respectively, for multiple comparisons. The same format is repeated in $\boldsymbol{D}-\boldsymbol{F}$ for within-periphery coherence and $\mathbf{G}-\boldsymbol{I}$ for center-periphery coherence.

2008; Wen et al., 2013; Seth et al., 2015). We analyzed Granger causal influences from the periphery to the center as FF GC influences, and influences from the center to the periphery as FB GC influences (Fig. 4). We found that both FF and FB GC were well above any bias level in the GC measure. FB GC appeared greater than FF in lower frequencies (Fig. 4A). However, as is apparent in Figure $4 A$, the variance of spectral GC influences also appears larger in lower frequencies. Thus, before comparing FF and FB GC we corrected for these statistical characteristics. To 

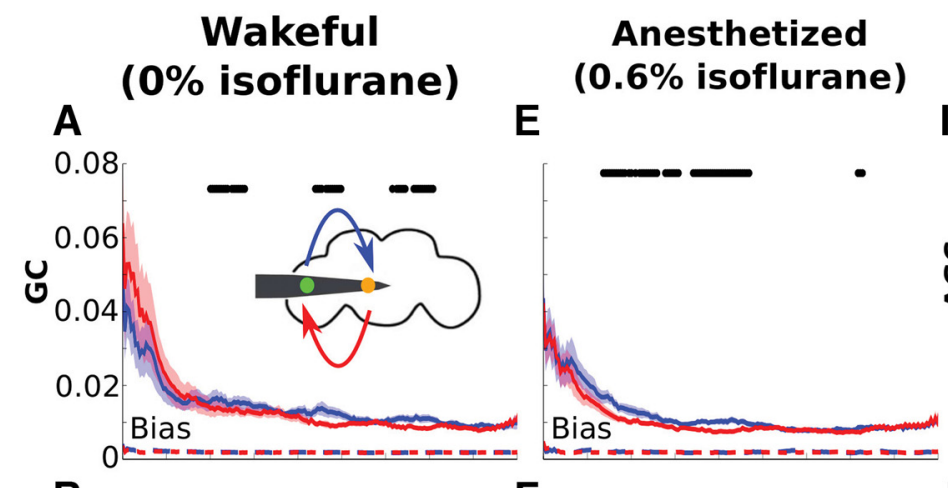

\section{Anesthetized-Wakeful (0.6\%-0\% isoflurane)}

I

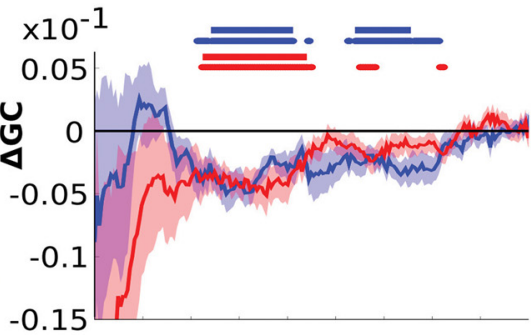

B

$F$

$\mathbf{J}$
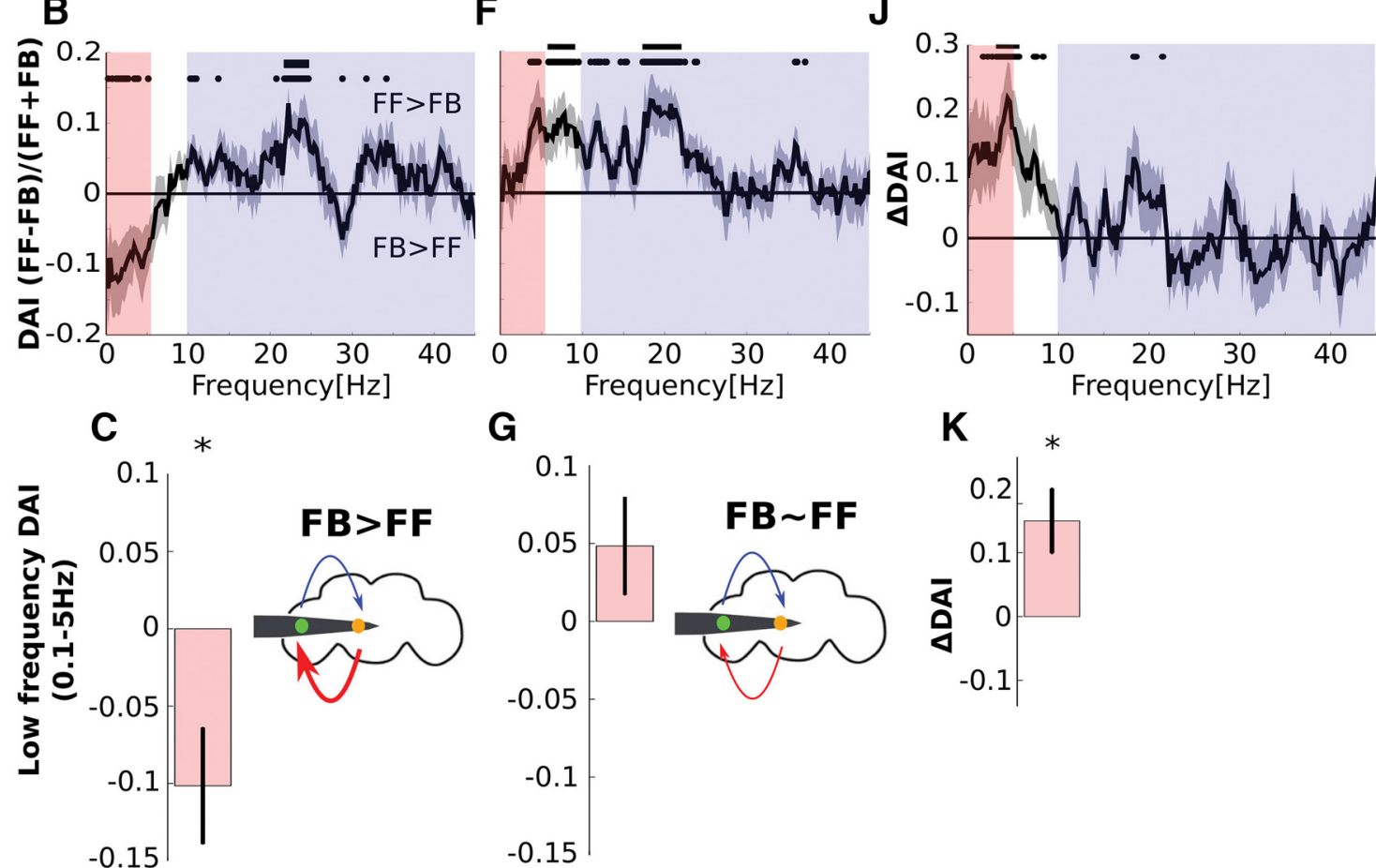

D

H
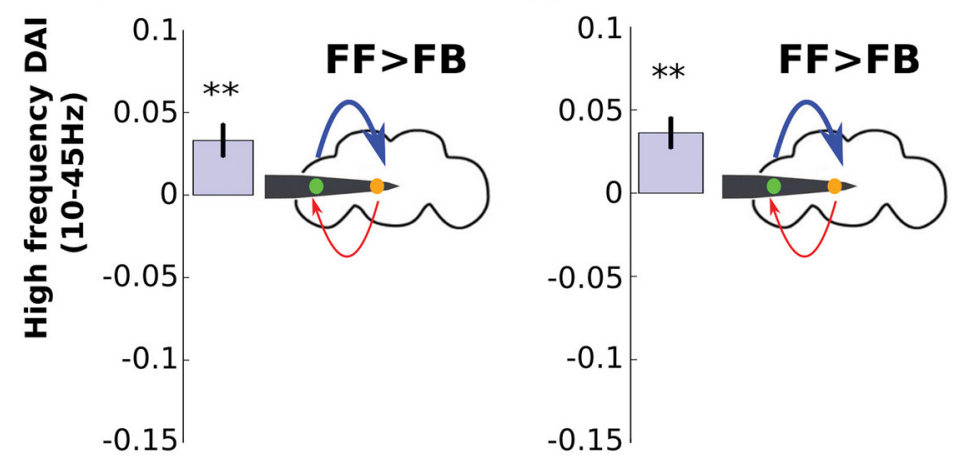

$\mathbf{L}$

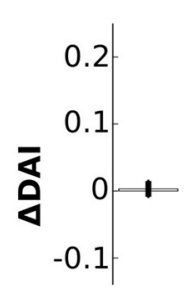

Figure 4. Isoflurane reduces low-frequency $(0.1-5 \mathrm{~Hz}) \mathrm{FB}$ in the fly brain. $\boldsymbol{A}$, Group level FF (blue) and FB (red) GC influences before any anesthesia ( $0 \%$ isoflurane). Black dots mark significant differences between FF and FB at the $p<0.05$ level (uncorrected). The FF and FB GC bias levels (see Materials and Methods) are shown as dashed red and blue lines, which almost completely overlap near zero. B, Group level DAI before any anesthesia ( $0 \%$ isoflurane). Positive values indicate FF $>$ FB and negative values indicate FF $<$ FB. Black dots and solid line mark significant uncorrected and corrected differences from zero at the $p<0.05$ level. Low $(0.1-5 \mathrm{~Hz})$ and high $(10-45 \mathrm{~Hz}$ ) frequencies are indicated by the pale pink and purple shadings. $\boldsymbol{C}, \boldsymbol{D}$, Group level DAl averaged for low (0.1-5 $\mathrm{Hz} ; \boldsymbol{C})$ and high $(10-45 \mathrm{~Hz} ; \boldsymbol{D})$ frequencies before any anesthesia ( $0 \%$ isoflurane). Negative and positive DAl values indicate that FB $>$ FF for low frequencies and that FB $<$ FF for high frequencies. The fly head schemas above the graphs depict this as thicker FF (red, $\boldsymbol{C}$ ) and FB (blue, $\boldsymbol{D}$ ) arrows. $\boldsymbol{E}-\boldsymbol{H}$, Same as $\boldsymbol{A}-\boldsymbol{D}$ for $0.6 \%$ isoflurane. $\boldsymbol{I}$, The effect isoflurane on FF (blue) and FB (red) GC, obtained by subtracting values in $0 \%$ isoflurane from $0.6 \%$ isoflurane. Blue (and red) dots and solid line mark significant uncorrected and 
continued

corrected reductions in FF (and FB) GC $(p<0.05)$. $J$, The effect of isoflurane on DAl, obtained by subtracting values in $0 \%$ isoflurane from $0.6 \%$ isoflurane. Black dots and solid line mark significant uncorrected and corrected differences from zero $(p<0.05)$. $\boldsymbol{K}, \boldsymbol{L}, \mathrm{T}$, The effect of $0.6 \%$ isoflurane on low $(\boldsymbol{K})$ and high frequencies $(\boldsymbol{L})$, obtained by subtracting values in $0 \%$ isoflurane $(\boldsymbol{C}, \boldsymbol{D})$ from $0.6 \%$ isoflurane $(\boldsymbol{G}, \boldsymbol{H}) . *$ and $* *$ indicate significant differences from zero at the $p<0.05$ and $p<0.01$ level, respectively (group-level permutation tests, see Materials and Methods). Error bars and shaded areas represent SEM across flies $(N=13)$ for all panels.

do this, we employed a normalized measure known as the DAl (Lee et al., 2013; Bastos et al., 2014; Michalareas et al., 2016). The DAl is defined as

$$
D A I=\frac{F F-F B}{F F+F B},
$$

which is positive when FF is stronger than $\mathrm{FB}$ and vice versa. For the DAl the variance was uniform across all frequencies (Fig. $4 B$ ).

During wakefulness ( $0 \%$ isoflurane), the DAl was negative for lower frequencies $(\sim 0.1-5 \mathrm{~Hz})$, indicating that $\mathrm{FB}$ is stronger than FF. For higher frequencies $(10-45 \mathrm{~Hz})$ the DAl is predominantly positive, indicating that FF is stronger than FB. To summarize these results, we averaged the DAl for low (0.1-5 Hz; Fig. 4C) and high (10-45 Hz; Fig. $4 D$ ) frequencies separately. We found that in awake flies $\mathrm{FB}$ is stronger than FF for low frequencies $(0.1-5 \mathrm{~Hz}$; Fig. $4 C, p<0.05$, group-level permutation tests, see Materials and Methods) and that FB is weaker than FF for high frequencies $(10-45 \mathrm{~Hz}$; Fig. $4 D, p<0.01)$. This global pattern of results, that is, the dominance of FB in lower frequencies and the dominance of $\mathrm{FF}$ in higher frequencies, is generally consistent with what has been reported in the awake mammalian brain (Bastos et al., 2014; Fontolan et al., 2014; van Kerkoerle et al., 2014; Michalareas et al., 2016).

\section{Isoflurane reduces FB}

Next, we examined the effect of isoflurane on FB and FF GC. In humans, general anesthetics have been shown to preferentially reduce FB (Lee et al., 2009; Boly et al., 2012; Jordan et al., 2013; Lee et al., 2013; Ranft et al., 2016). In our data, we found that during anesthesia both FF and FB influences were reduced in magnitude (Fig. 4E). The DAI, which normalizes the magnitude and variance across frequencies, was no longer negative for low frequencies but remained positive for high frequencies (Fig. 4F). This meant that during anesthesia the dominance of FB over FF was lost in low frequencies (Fig. 4G), and that the dominance of FF over FB in high frequencies remained intact (Fig. 4H). Statistical testing confirmed a frequencyand direction-specific effects on GC (Fig. 4/), resulting in an increase in the DAI in low frequencies (Fig. 4J,K; 0.1-5 $\mathrm{Hz}, p<0.05)$. There was no change in the DAl in high frequencies (Fig. $4 L ; 10-45 \mathrm{~Hz}, p=0.8$ ).

\section{Discussion}

In hierarchically organized brains, dedicated mechanisms may be necessary for routing information in an effective manner. In particular, the hallmark dynamics of hierarchical brains may be the modulation of lower-level, fast responses to the changing environment by higher- level, slower processing that incorporate prior information and expectation (Tani and Nolfi, 1999; Friston, 2010; Bastos et al., 2012; Hohwy, 2013). We found that, like human and monkey brains, FB and FF influences are mediated by lower and higher frequencies in fly brains. This result is surprising given the obvious differences in the number of neurons $\left[\sim 10^{5}\right.$ neurons in flies (Chiang et al., 2011) and $10^{11}$ neurons in humans (Herculano-Houzel, 2009)] and gross neuroanatomy between these brains. However, a recent study that analyzed the fly connectome has demonstrated a number of organizational similarities between the fly and mammalian brains, such as hierarchical structure and other graph theoretical characteristics (e.g., small-world and rich-club organization; Shih et al., 2015). These structural similarities may translate to similar dynamic characteristics of FB and FF, as recently demonstrated by analytical and computational studies (Moon et al., 2015, 2017; Mejias et al., 2016).

From a functional viewpoint, the dynamical characteristics of FF and FB may also serve similar roles across species, and possibly even hierarchically organized robots (Yamashita and Tani, 2008). Predictive coding suggests that "predictions" travel in the FB direction while "prediction errors" travel in the FF direction (Tani and Nolfi, 1999; Friston, 2010; Bastos et al., 2012; Hohwy, 2013). Because the updating of predictions depends on gathering prediction errors over time, predictions are inherently mediated through slower time scales (lower frequencies) than prediction errors (Bastos et al., 2012; Bastos et al., 2015; Michalareas et al., 2016). In insects, a number of recent studies have demonstrated predictive behaviors and begun to investigate their neural correlates (Card and Dickinson, 2008; Combes, 2015; Kim et al., 2015; Mischiati et al., 2015). Our finding that FB and FF influences are mediated by low and high frequencies in the fly brain is consistent with the idea that these animals are also engaging in a type of predictive coding. Predictive coding can be formulated under the more fundamental framework of free-energy minimization (Friston, 2010), which may apply to biological systems more broadly (Friston, 2012).

We found that isoflurane reduced both local coherence within the center and periphery as well as global coherence between the center and periphery. For directional connectivity, we found that both FF and FB were reduced, but that for low frequencies isoflurane preferentially reduced FB. A number of human studies have demonstrated that general anesthetics preferentially reduce frontal to posterior FB, as measured using EEG (Lee et al., 2009, 2013; Boly et al., 2012; Jordan et al., 2013; Ranft et al., 2016). Interestingly, a recent study using magneto- 
encephalography (MEG) found that such anterior to posterior (FB) influences are mediated by lower frequencies than those that mediated posterior to anterior (FF) influences (Hillebrand et al., 2016).

Our findings in flies parallel results previously reported for mammals. However, we acknowledge important limitations in regards to this comparison. In particular, we presented analyses of spontaneous, not sensory-evoked, LFPs. In the absence of a sensory signal to transmit, we do not know if the FF and FB we detected signal bottom-up and topdown processing. In this sense, cognitive processing or information transfer was not directly assessed. In Bastos et al. (2014), the presentation of a stimulus was necessary for clearly recruiting FF influences, which were interpreted as signaling bottom-up processing. While we did record data from periods of visual simulation (for details, see Cohen et al., 2016), these data were dominated by the stimulus frequency, violating the assumption of GC. It remains possible that in an experiment that uses sensory stimulation to engage bottom-up processes (yet left the data suitable for GC analysis), we would have detected more pronounced FF signaling and that this signaling would in fact be reduced by general anesthetics.

We also emphasize that not all general anesthetic effects found in mammals translate to flies. Indeed, one of the clearest system-wide effects of general anesthetics (as well as sleep) is an increase in low-frequency (0.1-4 $\mathrm{Hz}$ ) power (Steriade et al., 1993; Amzica and Steriade, 1998; Chauvette et al., 2011; Murphy et al., 2011; Lewis et al., 2012; Purdon et al., 2013, 2015). Such an increase has not been reported for insects (van Swinderen, 2006; Kirszenblat and van Swinderen, 2015), and we did not observe this here (the results did not change with longer time window (18 s) and/or using a unipolar reference scheme [data not shown]). Interestingly, a recent study reported that sleep induction in flies is accompanied by an increase in 7-10 Hz LFPs power (Yap et al., 2017). However, this was not observed for pharmacologically induced sleep, so any relevance to general anesthesia remains unknown. Further studies of population-level dynamics in flies will be required to fully understand the similarities between the way general anesthetics affect the fly and mammalian brain. Our study is a step in this direction, and suggests that low-frequency FB may be a system-wide target process for general anesthetics that is conserved across species.

\section{References}

Amzica F, Steriade M (1998) Electrophysiological correlates of sleep delta waves. Electroencephalogr Clin Neurophysiol 107:69-83. Medline

Barrett AB, Murphy M, Bruno M-A, Noirhomme Q, Boly M, Laureys S, Seth AK (2012) Granger causality analysis of steady-state electroencephalographic signals during propofol-induced anaesthesia. PLoS One 7. CrossRef

Bastos AM, Usrey MW, Adams RA, Mangun GR, Fries P, Friston KJ (2012) Canonical microcircuits for predictive coding. Neuron 76: 695-711. CrossRef Medline

Bastos AM, Litvak V, Moran R, Bosman CA, Fries P, Friston KJ (2015) A DCM study of spectral asymmetries in feedforward and feedback connections between visual areas V1 and V4 in the monkey. Neuroimage 108:460-475. CrossRef Medline
Bastos AM, Vezoli J, Bosman C, Schoffelen J-M, Oostenveld R, Dowdall J, Weerd P, Kennedy H, Fries P (2014) Visual areas exert feedforward and feedback influences through distinct frequency channels. Neuron 85:390-401.

Bendat JS, Piersol AG (2000) Random data: analysis and measurement procedures, Ed 3. New York: Wiley, Inc.

Boly M, Moran R, Murphy M, Boveroux P, Bruno M-A, Noirhomme Q, Ledoux D, Bonhomme V, Brichant J-F, Tononi G, Laureys S, Friston K (2012) Connectivity changes underlying spectral EEG changes during propofol-induced loss of consciousness. J Neurosci 32:7082-7090. CrossRef Medline

Card G, Dickinson MH (2008) Visually mediated motor planning in the escape response of Drosophila. Curr Biol 18:1300-1307. CrossRef

Chauvette S, Crochet S, Volgushev M, Timofeev I (2011) Properties of slow oscillation during slow-wave sleep and anesthesia in cats. J Neurosci 31:14998-15008. CrossRef Medline

Chen Y, Bressler SL, Ding M (2006) Frequency decomposition of conditional Granger causality and application to multivariate neural field potential data. J Neurosci Methods 150:228-237. CrossRef

Chiang AS, Lin CY, Chuang CC, Chang HM, Hsieh CH, Yeh CW, Shih CT, Wu JJ, Wang GT, Chen YC, Wu CC, Chen GY, Ching YT, Lee PC, Lin CY, Lin HH, Wu CC, Hsu HW, Huang YA, Chen JY, et al. (2011) Three-dimensional reconstruction of brain-wide wiring networks in Drosophila at single-cell resolution. Curr Biol 21:1-11. CrossRef Medline

Cohen D, Tsuchiya N (2017) A unified framework for dissecting the effects of common signals on functional and effective connectivity analyses: power, coherence, and Granger causality. https://www. biorxiv.org/content/early/2017/09/08/186122

Cohen D, Zalucki OH, van Swinderen B, Tsuchiya N (2016) Local versus global effects of isoflurane anesthesia on visual processing in the fly brain. eNeuro 3. CrossRef

Combes SA (2015) Neuroscience: dragonflies predict and plan their hunts. Nature 517:279-280. CrossRef Medline

Dhamala M, Rangarajan G, Ding M (2008) Estimating Granger causality from Fourier and Wavelet transforms of time series data. Phys Rev Lett 100:018701. CrossRef

Faes L, Pinna GD, Porta A, Maestri R, Nollo GD (2004) Surrogate data analysis for assessing the significance of the coherence function. IEEE Trans Biomed Eng 51:1156-1166. CrossRef

Fasoula A, Attal Y, Schwartz D (2013) Comparative performance evaluation of data-driven causality measures applied to brain networks. J Neurosci Methods 215:170-189. CrossRef Medline

Fontolan L, Morillon B, Liegeois-Chauvel C, Giraud A-L (2014) The contribution of frequency-specific activity to hierarchical information processing in the human auditory cortex. Nat Commun 5:4694. CrossRef Medline

Friston KJ (2010) The free-energy principle: a unified brain theory? Nat Rev Neurosci 11:127-138. CrossRef Medline

Friston KJ (2012) A free energy principle for biological systems. Entropy 14:2100-2121. CrossRef

Geweke JF (1982) Measurement of linear dependence and feedback between multiple time series. J Am Stat Assoc 77:304-313. CrossRef

Geweke JF (1984) Measures of conditional linear dependence and feedback between time series. J Am Stat Assoc 79:907-915.

Haberkern H, Jayaraman V (2016) Studying small brains to understand the building blocks of cognition. Curr Opin Neurobiol 37:5965. CrossRef Medline

Herculano-Houzel S (2009) The human brain in numbers: a linearly scaled-up primate brain. Front Hum Neurosci 3:31. CrossRef Medline

Hillebrand A, Tewarie P, van Dellen E, Yu M, Carbo EW, Douw L, Gouw AA, van Straaten EC, Stam CJ (2016) Direction of information flow in large-scale resting-state networks is frequencydependent. Proc Natl Acad Sci USA 113:3867-3872. CrossRef Medline

Hohwy J (2013) The predictive mind. Oxford: Oxford University Press. 
Hudetz AG, Mashour GA (2016) Disconnecting consciousness: is there a common anesthetic end point? Anesth Analg 123:12281240. CrossRef Medline

Imas OA, Ropella KM, Wood JD, Hudetz AG (2006) Isoflurane disrupts anterio-posterior phase synchronization of flash-induced field potentials in the rat. Neurosci Lett 402:216-221. CrossRef Medline

Ito $\mathrm{K}$, Shinomiya $\mathrm{K}$, Ito $\mathrm{M}$, Armstrong JD, Boyan G, Hartenstein V, Harzsch S, Heisenberg M, Homberg $U$, Jenett $A$, Keshishian $H$, Restifo LL, Rössler W, Simpson JH, Strausfeld NJ, Strauss R, Vosshall LB; Insect Brain Name Working Group (2014) A systematic nomenclature for the insect brain. Neuron 81:755-765. CrossRef

Jarvis MR, Mitra PP (2001) Sampling properties of the spectrum and coherency of sequences of action potentials. Neural Comput 13: 717-749. Medline

Jordan D, Ilg R, Riedl V, Schorer A, Grimberg S, Neufang S, Omerovic A, Berger S, Untergehrer G, Preibisch C, Schulz E, Schuster T, Schröter M, Spoormaker V, Zimmer C, Hemmer B, Wohlschläger A, Kochs EF, Schneider G (2013) Simultaneous electroencephalographic and functional magnetic resonance imaging indicate impaired cortical top-down processing in association with anesthetic-induced unconsciousness. Anesthesiology 119:1031. CrossRef Medline

Kim AJ, Fitzgerald JK, Maimon G (2015) Cellular evidence for efference copy in Drosophila visuomotor processing. Nat Neurosci 18:1247-1255. CrossRef Medline

Kirszenblat L, van Swinderen B (2015) The yin and yang of sleep and attention. Trends Neurosci 38:776-786. CrossRef Medline

Ku S-W, Lee U, Noh G-J, Jun I-G, Mashour GA (2011) Preferential inhibition of frontal-to-parietal feedback connectivity is a neurophysiologic correlate of general anesthesia in surgical patients. PLoS One 6. CrossRef

Lamme VA, Zipser K, Spekreijse H (1998) Figure-ground activity in primary visual cortex is suppressed by anesthesia. Proc Natl Acad Sci USA 95:3263-3268. Medline

Lee U, Kim S, Noh G-J, Choi B-M, Hwang E, Mashour GA (2009) The directionality and functional organization of frontoparietal connectivity during consciousness and anesthesia in humans. Conscious Cogn 18:1069-1078. CrossRef Medline

Lee U, Ku S, Noh G, Baek S, Choi B, Mashour GA (2013) Disruption of frontal-parietal communication by ketamine, propofol, and sevoflurane. Anesthesiology 118:1264. CrossRef Medline

Lewis LD, Weiner VS, Mukamel EA, Donoghue JA, Eskandar EN, Madsen JR, Anderson WS, Hochberg LR, Cash SS, Brown EN, Purdon PL (2012) Rapid fragmentation of neuronal networks at the onset of propofol-induced unconsciousness. Proc Natl Acad Sci USA 109:E3377-E3386. CrossRef

Littleton JT, Ganetzky B (2000) Ion channels and synaptic organization: analysis of the Drosophila genome. Neuron 26:35-43. Medline

Maksimow A, Silfverhuth M, Långsjö J, Kaskinoro K, Georgiadis S, Jääskeläinen S, Scheinin H (2014) Directional connectivity between frontal and posterior brain regions is altered with increasing concentrations of propofol. PLoS One 9. CrossRef

Maris E, Oostenveld R (2007) Nonparametric statistical testing of EEG- and MEG-data. J Neurosci Methods 164:177-190. CrossRef Medline

Mejias JF, Murray JD, Kennedy H, Wang X-JJ (2016) Feedforward and feedback frequency-dependent interactions in a large-scale laminar network of the primate cortex. Sci Adv 2:e1601335.

Michalareas G, Vezoli J, van Pelt S, Schoffelen J-M, Kennedy H, Fries $P$ (2016) Alpha-beta and gamma Rhythms subserve feedback and feedforward influences among human visual cortical areas. Neuron 89:384-397. CrossRef Medline

Mischiati M, Lin H-T, Herold P, Imler E, Olberg R, Leonardo A (2015) Internal models direct dragonfly interception steering. Nature 517: 333-338. CrossRef Medline
Mitra P, Bokil H (2007) Observed brain dynamics. Oxford: Oxford University Press.

Mitra PP, Pesaran B (1999) Analysis of dynamic brain imaging data. Biophys J 76:691-708. CrossRef Medline

Moon J-Y, Lee U, Blain-Moraes S, Mashour GA (2015) General relationship of global topology, local dynamics, and directionality in large-scale brain networks. PLoS Comput Biol 11. CrossRef

Moon JY, Kim J, Ko TW, Kim M, Iturria-Medina Y, Choi JH, Lee J, Mashour GA, Lee U (2017) Structure shapes dynamics and directionality in diverse brain networks: mathematical principles and empirical confirmation in three species. Sci Rep 7:46606. CrossRef Medline

Murphy M, Bruno M-A, Riedner BA, Boveroux $P$, Noirhomme $Q$, Landsness EC, Brichant J-F, Phillips C, Massimini M, Laureys S, Tononi G, Boly M (2011) Propofol anesthesia and sleep: a highdensity EEG study. Sleep 34:283. Medline

Nicolaou N, Hourris S, Alexandrou P, Georgiou J (2012) EEG-based automatic classification of 'awake' versus 'anesthetized' state in general anesthesia using Granger causality. PLoS One 7. CrossRef Oostenveld R, Fries P, Maris E, Schoffelen J-M (2011) FieldTrip: open source software for advanced analysis of MEG, EEG, and invasive electrophysiological data. Comput Intell Neurosci 2011: 1-9. CrossRef

Otsuna H, Ito K (2006) Systematic analysis of the visual projection neurons of Drosophila melanogaster. I. Lobula-specific pathways. J Comp Neur 497:928-958. CrossRef Medline

Pal D, Silverstein BH, Lee H, Mashour GA (2016) Neural correlates of wakefulness, sleep, and general anesthesia: an experimental study in rat. J Am Soc Anesthesiol 125:929-942. CrossRef

Papadopoulou M, Friston K, Marinazzo D (2015) Estimating directed connectivity from cortical recordings and reconstructed sources. Brain Topogr 1-12. CrossRef

Paulk AC, Millard SS, van Swinderen B (2013a) Vision in Drosophila: seeing the world through a model's eyes. Annu Rev Entomol 58:313-332. CrossRef Medline

Paulk AC, Kirszenblat L, Zhou Y, van Swinderen B (2015) Closedloop behavioral control increases coherence in the fly brain. $J$ Neurosci 35:10304-10315. CrossRef Medline

Paulk AC, Zhou Y, Stratton P, Liu L, van Swinderen B (2013b) Multichannel brain recordings in behaving Drosophila reveal oscillatory activity and local coherence in response to sensory stimulation and circuit activation. J Neurophysiol 110:1703-1721.

Purdon PL, Sampson A, Pavone KJ, Brown EN (2015) Clinical electroencephalography for anesthesiologists: part I: background and basic signatures. Anesthesiology 123:937-960. CrossRef Medline

Purdon PL, Pierce ET, Mukamel EA, Prerau MJ, Walsh JL, Wong KK, Salazar-Gomez AF, Harrell PG, Sampson AL, Cimenser A, Ching S, Kopell NJ, Tavares-Stoeckel C, Habeeb K, Merhar R, Brown EN (2013) Electroencephalogram signatures of loss and recovery of consciousness from propofol. Proc Natl Acad Sci USA 110: E1142-E1151. CrossRef

Ranft A, Golkowski D, Kiel T, Riedl V, Kohl P, Rohrer G, Pientka J, Berger S, Thul A, Maurer M, Preibisch C, Zimmer C, Mashour GA, Kochs EF, Jordan D, llg R (2016) Neural correlates of sevofluraneinduced unconsciousness identified by simultaneous functional magnetic resonance imaging and electroencephalography. Anesthesiology 125:861-872. CrossRef Medline

Sanes JR, Zipursky SL (2010) Design principles of insect and vertebrate visual systems. Neuron 66:15-36. CrossRef Medline

Seth AK (2010) A MATLAB toolbox for Granger causal connectivity analysis. J Neurosci Methods 186:262-273. CrossRef Medline

Seth AK, Barrett AB, Barnett $L$ (2015) Granger causality analysis in neuroscience and neuroimaging. J Neurosci 35:3293-3297. CrossRef Medline

Shih CT, Sporns O, Yuan SL, Su TS, Lin YJ, Chuang CC, Wang TY, Lo CC, Greenspan RJ, Chiang AS (2015) Connectomics-based analysis of information flow in the Drosophila brain. Curr Biol 25:1249-1258. CrossRef Medline 
Shirhatti V, Borthakur A, Ray S (2016) Effect of reference scheme on power and phase of the local field potential. Neural Comput 28: 882-913. CrossRef

Sonner JM (2008) A hypothesis on the origin and evolution of the response to inhaled anesthetics. Anesth Analg 107:849-854. CrossRef Medline

Steriade M, Nuñez A, Amzica F (1993) A novel slow ( $<1 \mathrm{~Hz}$ ) oscillation of neocortical neurons in vivo: depolarizing and hyperpolarizing components. J Neurosci 13:3252-3265. Medline

Tani J, Nolfi S (1999) Learning to perceive the world as articulated: an approach for hierarchical learning in sensory-motor systems. Neural Netw 12:1131-1141. Medline

Trongnetrpunya A, Nandi B, Kang D, Kocsis B, Schroeder CE, Ding $M$ (2016) Assessing Granger causality in electrophysiological data: removing the adverse effects of common signals via bipolar derivations. Front Syst Neurosci 9:189. CrossRef Medline

Truccolo WA, Ding M, Knuth KH, Nakamura R, Bressler SL (2002) Trial-to-trial variability of cortical evoked responses: implications for the analysis of functional connectivity. Clin Neurophysiol 113: 206-226. Medline

van Kerkoerle T, Self MW, Dagnino B, Gariel-Mathis M-AA, Poort J, van der Togt C, Roelfsema PR (2014) Alpha and gamma oscillations characterize feedback and feedforward processing in monkey visual cortex. Proc Natl Acad Sci USA 111:14332-14341. CrossRef

van Swinderen B (2006) A succession of anesthetic endpoints in the Drosophila brain. J Neurobiol 66:1195-1211. CrossRef Medline

van Swinderen B, Kottler B (2014) Explaining general anesthesia: a two step hypothesis linking sleep circuits and the synaptic release machinery. BioEssays 36:372-381. CrossRef Medline
Wang X, Chen Y, Ding M (2008) Estimating Granger causality after stimulus onset: a cautionary note. Neuroimage 41:767-776. CrossRef Medline

Wen X, Rangarajan G, Ding M (2013) Multivariate Granger causality: an estimation framework based on factorization of the spectral density matrix. Philos Trans A Math Phys Eng Sci 371:20110610. CrossRef

Wollstadt P, Sellers KK, Rudelt L, Priesemann V, Hutt A, Fröhlich F, Wibral M (2017) Breakdown of local information processing may underlie isoflurane anesthesia effects. PLoS Comput Biol 13: e1005511. CrossRef Medline

Wu M, Nern A, Williamson WR, Morimoto MM, Reiser MB, Card GM, Rubin GM (2016) Visual projection neurons in the Drosophila lobula link feature detection to distinct behavioral programs. Elife 5 . CrossRef

Yamashita Y, Tani J (2008) Emergence of functional hierarchy in a multiple timescale neural network model: a humanoid robot experiment. PLoS Comput Biol 4:e1000220. CrossRef

Yap MHW, Grabowska MJ, Rohrscheib C, Jeans R, Troup M, Paulk AC, van Alphen B, Shaw PJ, van Swinderen B (2017) Oscillatory brain activity in spontaneous and induced sleep stages in flies. Nat Commun 8:1815. CrossRef Medline

Zalucki OH, van Swinderen B (2016) What is unconsciousness in a fly or a worm? A review of general anesthesia in different animal models. Conscious Cogn 44:72-88. CrossRef Medline

Zhang X, Liu H, Lei Z, Wu Z, Guo A (2013) Lobula-specific visual projection neurons are involved in perception of motion-defined second-order motion in Drosophila. J Exp Biol 216:524-534. CrossRef Medline 